FORMATION Formation emploi

Revue française de sciences sociales

118 | avril-juin 2012

10 ans de parcours professionnels des jeunes :

l'intérêt des études longitudinales

\title{
Ségrégation professionnelle et salaires en début de carrière : regard sur quelques professions
}

Occupational gender segregation and wages in early careers: an insight on a few typical occupations

Berufliche Segregation und Entlohnung am Laufbahnbeginn: einige Berufe unter der Lupe

Segregación profesional y salarial al inicio de la carrera : una mirada sobre algunas profesiones

Thomas Couppié, Arnaud dupray et Stéphanie Moullet

\section{OpenEdition}

Journals

Édition électronique

URL : http://journals.openedition.org/formationemploi/3587

DOI : 10.4000/formationemploi.3587

ISSN : 2107-0946

Éditeur

La Documentation française

Édition imprimée

Date de publication : 30 juin 2012

Pagination : $37-59$

ISSN : 0759-6340

Référence électronique

Thomas Couppié, Arnaud dupray et Stéphanie Moullet, « Ségrégation professionnelle et salaires en début de carrière : regard sur quelques professions », Formation emploi [En ligne], 118 | avril-juin 2012, mis en ligne le 24 juillet 2012, consulté le 30 octobre 2020. URL : http://journals.openedition.org/ formationemploi/3587 ; DOI : https://doi.org/10.4000/formationemploi.3587 


\section{Ségrégation professionnelle et salaires en début de carrière :}

\section{Regard sur quelques professions}

Thomas CouppIÉ

Démographe, chargé d'études au Céreq, Deeva (Département entrées et évolutions dans la vie active)

Arnaud DupraY

Économiste, chargé d'études au Céreq, Deeva

(Département entrées et évolutions dans la vie active)

et chercheur associé au Lest (Laboratoire d'économie et de sociologie du travail)

Stéphanie Moullet

Économiste, maîtresse de conférence, Lest

(Laboratoire d'économie et de sociologie du travail) et université de la Méditerranée

Résumé

Ségrégation professionnelle et salaires en début de carrière : regard sur quelques professions

Cet article vise à éclairer l'évolution de la ségrégation professionnelle selon le sexe, au cours des dix premières années de carrière, et ses liens avec les écarts de salaire. Au bout des dix ans, cette ségrégation a peu augmenté, la part des professions dont la ségrégation est d'origine scolaire s'est réduite et l'écart salarial s'est en moyenne accru. Centrée sur quelques professions emblématiques, l'analyse montre que la situation salariale des femmes se dégrade. Toutefois, les liens entre ségrégation professionnelle et discrimination salariale des femmes ne sont pas univoques.

Mots clés : Division sexuelle du travail $\bullet$ inégalité salariale $\bullet$ femme $\bullet$ infirmier $\bullet$ profession intermédiaire $\bullet$ enseignant $\bullet$ métier de la sécurité

Abstract

Occupational gender segregation and wages in early careers: an insight on a few typical occupations

This paper aims at assessing occupational gender segregation evolution during early careers and its links with gender wage gap. Among people having experienced ten years 
on the labour market, occupational gender segregation has slightly increased, occupations characterized by an educative-led segregation have a reduced impact, and the wage gap has increased on average. Focusing on a few typical and revealing occupations, the analysis shows that women wage situation worsens. However, links between occupational gender segregation and female wage discrimination differ across occupations.

Key words: Gender based division of labour • wage inequality • woman $\bullet$ nurse • intermediate occupation $\bullet$ teacher $\bullet$ occupation in security

Journal of Economic Literature: J 24, J 16, J 31

Traduction : Auteurs

Depuis deux décennies, des travaux portant sur les écarts salariaux entre hommes et femmes s'attachent à évaluer, de manière théorique ou empirique, l'impact de la composition sexuée des professions sur les différentiels de gains (Groshen, 1991 ; Reskin, 1993 ; Macpherson et Hirsch, 1995 ; Baker et Fortin, 2001 ; Bayard, Hellerstein, Neumark et Troske, 2003 ; Tomaskovic-Devey et Skaggs, 2002, etc.). Dans la littérature française, les relations entre la nature des écarts de salaire observés entre hommes et femmes et les caractéristiques des différentes professions occupées ne sont que depuis peu abordées (Meng et Meurs, 2001 ; Couppié, Dupray et Moullet, 2006). Cette dernière étude a permis notamment de montrer que l'écart salarial en début de vie active varie selon « le genre » de la profession (et donc le degré de ségrégation qui la caractérise), mais aussi selon l'origine de la ségrégation ; cette dernière pouvant être plutôt " héritée » de la ségrégation éducative (la profession recrutant dans des formations sexuellement clivées) ou plutôt le fruit des mécanismes d'appariement s'opérant sur le marché du travail entre individus et emplois sexuellement différenciés, indépendamment des qualifications scolaires acquises (Couppié et Epiphane, 2004a).

Grâce à la quatrième enquête du Céreq sur la Génération 98, nous disposons désormais non seulement des informations sur les emplois occupés par cette génération en 2001 mais aussi sur ceux détenus après dix années de vie professionnelle.

Ces données nous permettent de poursuivre, à travers cette contribution, un double objectif. Le premier est d'évaluer l'évolution de la ségrégation professionnelle au cours des débuts de carrière avec deux questions corrélatives : la part des professions dont la ségrégation est principalement d'origine scolaire se resserre-t-elle ou au contraire s'accroîtelle avec des réorientations professionnelles plus en cohérence avec les investissements éducatifs initiaux ? Dans quelle mesure ces évolutions concourent aux niveaux de salaire observés?

Une fois décrite la nature des évolutions pour l'ensemble des professions, le second objectif de l'article est d'analyser l'évolution des différentiels de salaire entre hommes et femmes au sein de quelques professions prises en exemple. On retient cinq professions qui se 
distinguent par leur composition sexuée en début de vie professionnelle, certaines emblématiques à cet égard, et parce qu'elles comportent suffisamment d'observations pour permettre de dégager l'effet salarial net du sexe au travers d'équations de gains. L'intérêt de l'analyse séparée réside notamment dans le fait que les évolutions des compositions sexuées et des écarts de salaires ne sont pas homogènes entre professions. Il s'agit donc d'apprécier comment la pénalisation salariale des femmes s'amplifie ou s'amenuise selon que la profession à laquelle elles appartiennent se compose et se recompose en matière sexuée; et ce, au gré des mobilités professionnelles des hommes et des femmes. Nous nous limiterons ici à une approche descriptive de ces dimensions.

Nous aborderons d'abord les débats autour du lien entre ségrégation professionnelle et inégalités salariales et la méthode retenue ici pour appréhender la ségrégation professionnelle. La deuxième partie sera consacrée aux évolutions, contrastées, de la ségrégation professionnelle et des écarts de salaires au cours des dix premières années de vie active. En développant les cas de cinq professions aux contextes organisationnels très différents, la dernière partie s’attachera à expliciter l'hétérogénéité de ces évolutions selon le type de profession.

\section{Appréhender les ségrégations}

La relative séparation des hommes et des femmes dans les emplois est un phénomène observé de longue date. Nous rappellerons d'abord les discussions sur la façon dont cette ségrégation professionnelle pourrait contribuer aux inégalités de salaires. Puis nous exposerons succinctement la manière dont nous avons mesuré, dans cette étude, la ségrégation professionnelle entre hommes et femmes.

\subsection{Ségrégation professionnelle et écarts salariaux : un tour d'horizon de la littérature}

La ségrégation des professions selon le genre n'est pas un fait nouveau ; cependant, on assiste à une lente réduction de celle-ci avec la montée de l'activité des femmes depuis trente ans et des changements concomitants dans les comportements à la fois des femmes et des employeurs. Les premières élargissent leur espace de formation et sont de plus en plus présentes dans des filières traditionnellement dominées par les hommes alors que les seconds deviennent plus ouverts à la présence de femmes dans des métiers où elles demeuraient absentes ou l'exception. La concentration des femmes dans l'emploi reste néanmoins importante : ainsi en 2002, dix familles professionnelles, parmi une nomenclature en comptant 84 , concentraient plus de la moitié des femmes actives occupées (Okba, 2004). Dans la plupart des pays de l'OCDE (Organisation de coopération et de développement économiques), coexistent une ségrégation verticale relative au fait que 
les femmes sont sous-représentées parmi les professions supérieures et d'encadrement, et une ségrégation horizontale - les femmes sont plus souvent concentrées dans certains domaines professionnels (Anker, 1998).

Ces aspects distributionnels sont alors susceptibles de peser sur l'écart des salaires selon le sexe. Par exemple, la fermeture aux femmes des professions les plus prestigieuses reporte leurs candidatures vers un nombre limité d'emplois ; ceci abaisse mécaniquement leur niveau de salaire par la loi de l'offre et de la demande (Sorensen, 1990).

Différentes approches théoriques ont été développées pour expliquer les écarts de situations salariales dus à ces aspects distributionnels. Les écarts de gains entre hommes et femmes au sein d'une même profession n'étant plus, de l'avis de certains spécialistes, une source importante des disparités salariales entre hommes et femmes (Petersen et Saporta, 2004).

- Pour la théorie du capital humain (Polachek, 1981 ; Mincer et Ofek, 1982), les femmes se concentrent dans les emplois où les risques de déperdition de capital humain dus aux interruptions de carrières sont les plus faibles. Les femmes feraient donc plus volontiers le choix de professions où à la fois les risques d'obsolescence technique du capital humain et les opportunités d'accroissement de celui-ci sont plus limités. Autant d'éléments qui pèseraient sur leur progression de carrière comparativement à celle de leurs homologues masculins. Ces explications axées sur la rationalité des choix féminins ont pourtant été battues en brèche dès les années 80, avec les travaux d'England (1982) et d'England et alii (1988), mais sont plutôt confirmées dans le cas Suédois par Hansen et Wahlberg (2008).

- Toujours dans une perspective mettant en exergue les préférences individuelles, Filer (1983) montre que des préférences inobservées (telles que les goûts individuels ou la personnalité) peuvent orienter des femmes vers des métiers comportant certaines caractéristiques bénéfiques, en contrepartie d'une moindre rémunération, tableau qui concorde avec la théorie des différences compensatrices de Killingsworth (1987). Celle-ci souligne que, dans l'appréciation de la valeur des emplois, des préférences pour des avantages non salariaux (tels que la sécurité d'emploi) peuvent compenser, inégalement chez les hommes et les femmes, un niveau de salaire inférieur. Encore faudrait-il établir qu'à profession donnée, hommes et femmes sont également concernés.

- Une autre approche plus sociologique est celle de la dévaluation des métiers féminins. Elle part des stéréotypes et représentations sociales du métier et de la qualification construits dans un cadre de division traditionnelle des rôles sexués. Ici, l'homme est vu comme principal pourvoyeur de ressources monétaires dans la sphère de la production et les tâches relatives à la sphère de la reproduction sont dévolues à la femme. Ils tendent à discréditer les compétences mises en œuvre dans les professions féminines (Steinberg, 1990 ; England, 1992 ; Daune-Richard, 1998). Une tendance à leur " naturalisation " conduirait à amoindrir leur reconnaissance salariale. 
Dans cette approche de la dévaluation des compétences mises en œuvre dans les professions féminisées, le taux important de femmes y ferait baisser le salaire moyen observé, indépendamment du niveau moyen de capital humain détenu par ses titulaires. Cette interprétation s'accorde avec l'observation que les femmes se trouvent plutôt concentrées dans des métiers de services où les protections collectives (convention collective, représentation syndicale) sont moindres que dans l'industrie, mais également affectées à des postes dont les contenus sont moins codifiés et plus mouvants que dans les métiers masculins (Lemière et Silvera, 2010).

- La ségrégation professionnelle pourrait également trouver sa source dans une discrimination de nature allocative, pour reprendre l'expression de Petersen et Saporta (2004). Deux mécanismes théoriques pourraient l'expliquer : la discrimination par goût (Becker, 1971) ou la discrimination statistique (Aigner et Cain, 1977). Pour la première, la prévention latente ou exprimée par des collectifs de travail masculins à l'encontre de l'intégration de femmes engendre une pression sur les employeurs qui limiterait de ce fait les embauches de candidates. Ce mécanisme soutiendrait une relative ségrégation professionnelle au sein des entreprises (Cockburn, 1991). La mixité du personnel serait acceptée au prix d'une ségrégation des postes selon le genre et d'une hiérarchie salariale entre métiers " cœurs ", à dominante masculine, et métiers "supports ", largement ouverts aux femmes. Dans ces conditions, en l'absence de mobilité, les disparités de salaire auraient peu de chance de disparaître avec l'expérience et les situations salariales individuelles seraient marquées avant tout par la hiérarchie salariale des professions.

- La théorie de la discrimination statistique explique quant à elle l'écart de salaire des hommes et des femmes en début de vie active en raison d'incertitudes spécifiques des employeurs quant à la productivité des femmes, leur attachement à l'emploi ou leurs signaux de productivité. Ces perceptions " en moyenne " se répercuteraient alors dans des affectations à des positions d'emploi spécifiques, moins prestigieuses et moins rémunérées que celles des hommes (Bielby et Baron, 1986). Le moindre salaire consenti à ces dernières relèverait ainsi d'un mécanisme assurantiel où l'incertitude initiale sur la productivité individuelle doit être provisionnée dans l'attente de nouvelles évaluations avec l'expérience. Dans ce cadre, la concentration des femmes dans certains métiers, moins valorisés du point de vue salarial mais non directement comparables dans leur contenu à ceux exercés par les hommes, permettrait à l'employeur d'instaurer un différentiel de traitement tout en limitant la présomption de comportement discriminatoire.

- À ce titre, pouvoir distinguer la ségrégation professionnelle selon qu'elle est le reflet de la ségrégation éducative ou qu'elle se construit en fonction de la conduite des employeurs est potentiellement fécond. En effet, un niveau important de ségrégation liée au marché du travail révèle une interaction a priori plus forte de caractéristiques non productives, ou de caractéristiques productives bien plus difficilement objectivables que le diplôme, dans les processus de recrutement. Cette dernière composante de la ségrégation devrait donc être révélatrice des possibilités de différenciation salariale dans le métier en début 
de vie professionnelle. On s'attendrait alors à ce qu'un niveau élevé de ségrégation professionnelle résultant des appariements sur le marché du travail soit associé d'une part, ou à une relative faiblesse du niveau moyen de salaire au sein des professions féminisées, ou, à l'inverse, à un niveau moyen élevé de salaire au sein des professions à dominante masculine ; d'autre part, à des écarts de salaire entre hommes et femmes potentiellement plus importants, une fois contrôlées les caractéristiques productives observables des individus. Dans cette logique, l'effet propre de la composition sexuée des professions sur le salaire des femmes devrait s'atténuer avec l'expérience professionnelle, et en particulier l'ancienneté dans l'emploi, à mesure que l'arrivée de nouveaux signaux individuels de productivité permet aux employeurs de réviser leurs évaluations initiales et d'aligner les salaires féminins sur des critères de productivité et de performance comparables à ceux de la maind'œuvre masculine. On serait face à une discrimination de nature allocative et transitoire destinée à s'estomper avec le temps.

Si les écarts de salaire au contraire s'amplifient avec l'expérience au sein d'une telle profession ségréguée, il faudrait en conclure que l'allocation initiale définit d'inégales opportunités de promotion et de développement de carrière pour les individus. Une partie des opportunités ultérieures de progression serait en quelque sorte encadrée par les conditions initiales de valorisation salariale dans la profession exercée à l'embauche.

Au plan empirique, nombre d'études ont tenté d'évaluer si le salaire variait en fonction de la composition sexuée de la profession. Au vu des travaux récents réalisés sur différents pays (Groshen, 1991 ; Macpherson and Hirsh, 1995 ; Bayard, Hellerstein, Neumark and Troske, 2003 ; Ruijter, Doorne-Huiskes et Schippers, 2003 ; Hansen et Wahlberg, 2008 ...), il apparaît que travailler dans une profession féminisée est associé à des niveaux de salaire inférieurs à ce qu'on trouve ailleurs. Plusieurs études montrent que cet effet est plus pénalisant pour les hommes que pour les femmes: Baker et Fortin (2001) dans le cas du Canada, de Ruijter et Huffman (2003) dans le cas des Pays-Bas. À l'inverse, Cohen et Huffman (2003), pour les États-Unis, montrent que l'écart salarial s'accroît selon le degré de féminisation de la profession. Busch et Holst (2011), dans le cas de l'Allemagne et des fonctions d'encadrement, observent que seules les femmes sont pénalisées en termes de salaire par la féminisation de la profession. Sur les tout débuts de vie active, Couppié, Dupray et Moullet (2006) ont mis en évidence que c'était moins la part des femmes dans une profession en tant que telle que sa combinaison avec l'origine de la ségrégation qui alimentait la moins-value salariale enregistrée par les femmes dans cette profession ${ }^{1}$.

Dans le cas de la France, et en se focalisant sur les débuts de vie professionnelle, on peut s'attendre à ce que le caractère féminisé d'une profession desserve aussi bien la situation salariale des hommes que celle des femmes, notamment si la valeur salariale est attachée à la profession exercée plus qu'à l'individu qui l'occupe ; alors que la ségrégation résul-

1. Toutefois, ces analyses étaient menées sur des regroupements de professions et non au niveau détaillé - en 77 postes - des familles professionnelles, tel qu'envisagé ici. 


\section{Encadré 1}

\section{La Génération 98 et ses ré-interrogations}

La Génération 98 a été interrogée par le Céreq (Centre d'études et de recherche sur les qualifications) à quatre reprises, en 2001, 2003, 2005 et 2008. Est interrogée, à chacune de ces dates, une population de jeunes sortis du système éducatif en 1998, représentative de l'ensemble des sortants de tous niveaux et spécialités de formation initiale en France métropolitaine. II s'agit d'une enquête rétrospective, les individus interrogés décrivant mois par mois leur situation (emploi, chômage, inactivité...) depuis leur sortie de formation initiale jusqu'à la date d'interrogation. Le premier emploi obtenu après la sortie du système éducatif de même que l'emploi décrit aux dates d'interrogation sont l'objet d'une information détaillée.

La quatrième interrogation, au printemps 2008, permet ainsi d'élargir la fenêtre d'observation au-delà de l'entrée dans la vie active et d'éclairer les débuts de carrière en essayant de saisir certains déterminants qui président à leur différenciation. À cette étape, l'échantillon est un peu inférieur à 11000 individus contre près de 55000 jeunes enquêtés en première vague. Nous définissons notre population d'analyse à partir de la dernière interrogation pour obtenir un quasi-cylindrage de l'échantillon sur la période.

tant des comportements des acteurs sur le marché du travail, indépendamment de l'offre sexuée d'éducation, pourrait davantage pénaliser spécifiquement les femmes. En effet, les professions dans lesquelles les recrutements révèlent une polarisation des sexes nettement supérieure à la répartition sexuée attendue du fait de l'offre éducative qui les alimente montrent la relative faiblesse du rôle des caractéristiques productives objectivables (tel que le diplôme) dans les décisions de recrutement ; de ce fait, elles seraient plus susceptibles de révéler des pratiques discriminatoires des employeurs à l'encontre des femmes.

\subsection{Les données et indicateurs de ségrégation}

\section{- Les données de cadrage}

Notre contribution porte sur une population des 9503 jeunes en emploi à la première interrogation de la Génération 98 ( $c f$. encadré $\mathbf{1}$ ), trois ans après la sortie du système éducatif, et de 9983 individus actifs occupés lors de la quatrième et dernière interrogation, en 2008. La population féminine représente $48 \%$ de la population étudiée, en 2001 comme en 2008.

Les salaires des individus sont exprimés en euros courants ; ils correspondent à la rémunération mensuelle nette, primes incluses ${ }^{2}$. En moyenne, le salaire des hommes dépasse celui des femmes de plus de $17 \%$ en 2001 et ce différentiel s'accroît pour atteindre, en 2008, plus de $21 \%$ ( $c f$. tableau 1). L'écart augmente sur la période à tous les niveaux de la distribution.

2. L'absence d'information sur le nombre effectif d'heures de travail ne nous permet pas de calculer des taux de salaire horaire. 
Tableau 1

Caractéristiques des hommes et des femmes en emploi

3 et 10 ans après la sortie du système éducatif (euros courants, \% ou effectifs selon colonne)

\begin{tabular}{|l|c|c|c|c|}
\hline \multirow{2}{*}{} & \multicolumn{2}{|c|}{ En 2001 } & \multicolumn{2}{c|}{ En 2008 } \\
\cline { 2 - 5 } & Hommes & Femmes & Hommes & Femmes \\
\hline Salaires & & & & \\
\hline \multicolumn{1}{|c|}{ Moyenne } & 1708 & 1455 & 1972 & 1625 \\
\hline Q1 (1er quartile) & 1116 & 998 & 1400 & 1185 \\
\hline Médiane & 1450 & 1300 & 1696 & 1475 \\
\hline Q3 (3eme quartile) & 1925 & 1677 & 2167 & 1805 \\
\hline Part emploi dans le public & 17 & 34 & 17 & 36 \\
\hline Part de CDI (contrat à durée indéterminée) ou fonctionnaire & 72 & 66 & 92 & 90 \\
\hline Part de temps plein & 91 & 82 & 94 & 71 \\
\hline Emploi avec encadrement d'1 à 5 subordonnés & 18 & 15 & 24 & 16 \\
\hline Emploi avec encadrement de plus de 5 subordonnés & 5 & 5 & 13 & 8 \\
\hline Nombre moyen d'années d'éducation & 12.9 & 13.6 & 12.9 & 13.6 \\
\hline Effectif & 4941 & 4562 & 5192 & 4791 \\
\hline
\end{tabular}

Source : Génération 98, interrogations 2001, 2008.

Champ : Jeunes en emploi.

L'écart salarial moyen est de $13 \%$ si seule est prise en compte la population travaillant à temps plein. Cet écart reste inchangé entre les situations trois ans et dix ans après la sortie du système éducatif. Pour autant, les femmes travaillent moins souvent que les hommes à temps plein en début de vie active mais surtout leur temps de travail se réduit bien plus fortement que pour leur condisciples avec l'arrivée des enfants ; $17 \%$ des femmes passent à temps partiel à l'arrivée du premier enfant (Epiphane, Gasquet et Moullet, 2008). Dix années après leur entrée sur le marché du travail, deux tiers des femmes sont mères et, parmi elles, moins de $62 \%$ occupent un emploi à temps plein (pour $94 \%$ des pères).

D'autres caractéristiques d'emploi distinguent les femmes des hommes. En proportion, les femmes sont deux fois plus souvent en emploi dans le secteur public : après dix années passées sur le marché du travail, ce secteur compte $36 \%$ des emplois féminins contre seulement $17 \%$ des emplois masculins. En outre, en matière de responsabilités hiérarchiques, l'écart entre les sexes se creuse dans le déroulement de la carrière : en 2008, avec près de six années d'ancienneté accumulée chez leur employeur de 2008 et plus de trois années d'expérience antérieure en moyenne, seulement $24 \%$ des femmes déclarent encadrer au moins un autre salarié contre $36 \%$ des hommes.

Enfin, parmi les autres déterminants salariaux, la formation initiale est considérée à l'aune de la durée de scolarité. Cette dernière est définie comme le nombre d'années néces- 
saires pour atteindre le plus haut diplôme obtenu à partir de l'âge de 6 ans. Les femmes obtiennent en moyenne des diplômes plus élevés que les hommes et passent ainsi, toujours en moyenne, près d'une année de plus dans le système éducatif.

Outre leurs caractéristiques individuelles propres, les jeunes enquêtés ont entamé leur vie professionnelle dans des professions qui ne sont pas toutes neutres au regard de la proportion d'hommes et de femmes qui y accèdent. La ségrégation de certaines professions pourrait ainsi affecter le salaire de leurs occupants, indépendamment de leurs caractéristiques productives propres.

\section{- Évaluer la ségrégation professionnelle}

De manière simple, la ségrégation professionnelle peut être évaluée par la proportion d'hommes (ou femmes) qui travaillent dans une profession ou un ensemble donné de professions, par rapport à leur représentation dans l'ensemble de la population. Pour la population des jeunes en début de vie active que l'on étudie, où les femmes représentent $48 \%$ de la cohorte, l'absence de ségrégation signifie que dans chaque profession ou groupe professionnel, la main-d'œuvre serait composée précisément de cette proportion de femmes ( $c f$. tableau 2).

Létat de la ségrégation observée à un moment du temps n'en révèle pas les causes. D'une part, les préférences ou les aspirations en matière de choix éducatifs et les possibilités de réalisation de ces choix, pour partie spécifiques à chaque sexe, peuvent être à l'origine de la ségrégation professionnelle. D'autre part, une fois le parcours scolaire achevé, la ségrégation peut également se développer au travers des mécanismes d'allocation dans les différents emplois et au travers des processus de recrutement menés par les employeurs.

En d'autres termes, l'inégale répartition des hommes et des femmes dans les professions trouve à la fois sa source dans le capital scolaire différencié des unes et des autres, dans les préférences et aspirations professionnelles des individus, et dans des mécanismes ségrégatifs d'allocation sur le marché du travail, à capital scolaire donné. Les préférences individuelles et les projets professionnels ne sont pas disponibles dans les données; dès lors, on suppose que les composantes du niveau d'éducation atteint (filière, spécialité, diplôme) intègrent pour partie ces préférences.

En partant de travaux précédents sur la ségrégation professionnelle, de Couppié et Epiphane $(2006$; 2009), on peut ainsi définir 77 groupes professionnels caractérisés par leur degré de ségrégation et l'origine de celle-ci. Trois indices de ségrégation sont définis et déclinables pour chacun des groupes professionnels : un indice général décomposé en deux sous-indices additifs, représentant respectivement la ségrégation d'origine éducative et celle qui prend corps sur le marché du travail ${ }^{3}$.

3. On renvoie le lecteur à Couppié et Epiphane $(2006,2009)$ pour une présentation détaillée des calculs des indicateurs de ségrégation. 
Tableau 2

Ségrégation professionnelle et origine de la ségrégation

\begin{tabular}{|l|c|c|}
\hline Expérience sur le marché du travail & $\mathbf{3}$ ans & $\mathbf{1 0}$ ans \\
\hline Part des femmes & $48,3 \%$ & $48,3 \%$ \\
\hline Indice de ségrégation $\left(^{*}\right):$ & 0,237 & 0,246 \\
\hline$\ldots$ d'origine éducative & 0,185 & 0,175 \\
\hline ... liée au marché du travail & 0,09 & 0,11 \\
\hline Part de la ségrégation liée au marché du travail / ségrégation totale & $28,4 \%$ & $33,7 \%$ \\
\hline Nombre de groupes professionnels & 77 & 77 \\
\hline Effectif & 9503 & 9983 \\
\hline
\end{tabular}

Source : Génération 98, interrogations 2001, 2008.

Champ : Jeunes en emploi.

$\left(^{*}\right)$ : L'indice de ségrégation total présenté dans ce tableau correspond à la moyenne pondérée des indices en valeur absolue par profession.

L'indice de ségrégation totale prend des valeurs dans l'intervalle $[0 ; 0,5]$ pour une cohorte équilibrée selon le sexe. La ségrégation est à son maximum pour la valeur maximale, ce qui signifie que la moitié de la population devrait changer de profession pour parvenir à un état de ségrégation nulle. Les tableaux $\mathrm{B}$ et $\mathrm{C}$, non reproduits ici, et disponibles au format électronique sur CAIRN (http://www.cairn.info/revue-formation-emploi.htm) fournissent la liste des professions et indicateurs de ségrégation associés.

L'intensité de la ségrégation professionnelle et son évolution pour l'ensemble des groupes professionnels sont d'abord exposées avant que soit examinée plus en détails la situation dans cinq professions.

\section{La ségrégation professionnelle s'accroit légèrement, les écarts de salaires se creusent fortement}

Après dix années passées sur le marché du travail, la ségrégation totale évolue peu, mais cette relative stabilité cache des évolutions plus importantes d'une profession à l'autre et quant au poids relatif de chacune de ses composantes. À l'inverse, l'évolution de l'écart de salaires entre hommes et femmes est nettement plus affirmée, au détriment des femmes. 


\subsection{La ségrégation liée au marché du travail augmente avec le déroulement des carrières}

À l'échelle des 77 groupes professionnels ${ }^{4}$ distingués ici, la ségrégation professionnelle évolue peu sur les débuts de carrière, puisqu'elle s'élève d'environ un point de pourcentage en sept ans. Cependant, cette faible augmentation correspond à des changements significatifs à la fois des taux de ségrégation et des professions concernées.

On peut définir le caractère plus ou moins sexué des professions selon la proportion de femmes qui les caractérise. Par convention, les professions dites masculines accueillent moins de $33 \%$ de femmes et les professions féminines plus de $63 \%$ de femmes. Les autres professions sont alors considérées comme mixtes 5 . En 2001 comme en 2008, on compte ainsi 30 professions à prédominance masculine, 24 professions féminisées et 23 groupes mixtes; ce qui n'exclut pas des changements d'appartenance des professions sur la période. Moins nombreuses, les professions féminines rassemblent pourtant, après dix ans de vie active comme après trois ans, une plus grande part de la population en emploi que les professions masculines, $40,5 \%$ vs. $38,1 \%$ en 2001 . Toutefois, professions féminines comme masculines perdent un peu en effectif au cours de cette période de sept ans, au profit des professions mixtes.

Suite aux mobilités professionnelles individuelles et aux évolutions de recrutements dans les différentes professions sur la période, l'origine de la ségrégation évolue également. Lincidence de la ségrégation d'origine éducative s'amenuise avec le temps alors que la ségrégation qui prend corps sur le marché du travail augmente. En calculant un indicateur qui rapporte la ségrégation liée au marché du travail à la ségrégation totale, on constate que cette part augmente de plus de cinq points entre 2001 et 2008. Une partition en trois classes des professions selon les valeurs de cet indicateur ${ }^{6}$ - moins de $25 \%$, de 25 à moins $50 \%$ et $50 \%$ et plus - permet d'apprécier plus finement les modalités de développement de cette composante de la ségrégation professionnelle entre sexes. Ainsi, en 2001, 16 professions étaient concernées par une ségrégation dont la majeure partie se construit sur le marché du travail, ensemble qui passe à 21 en 2008. La première catégorie, celle dont l'influence de la ségrégation liée au marché du travail est faible, perd un nombre de professions ; cette perte est équivalente à l'accroissement constaté dans la troisième catégorie.

4. Par commodité, dans les commentaires on parlera de professions, étant entendu que l'on se réfère aux groupes professionnels identifiés ici.

5. Ceci correspond à un intervalle de $+-15 \%$ autour de la moyenne des femmes dans l'échantillon. Ces frontières pour définir la féminisation ou la masculinisation d'une profession restent une affaire de convention. Cet aspect a été discuté dans Couppié, Dupray, Moullet (2011).

6. On écarte les professions mixtes du raisonnement car, pour les professions peu ségrégées, l'analyse de l'origine de la ségrégation a moins de sens. 
Le poids de la ségrégation professionnelle d'origine éducative tend donc à s'effacer pour un nombre croissant de groupes professionnels, au profit des préférences et opportunités des employeurs et salariés.

Enfin, notons que parmi les professions dans lesquelles la ségrégation liée au marché du travail a une incidence relative faible, certaines sont marquées, en 2001 comme en 2008, par une ségrégation sur le marché du travail qui s'oppose à celle produite par l'éducation. Le recrutement des techniciens médicaux s'opère plutôt, à formation identique, en faveur des hommes, en 2001 comme en 2008, mais la profession fait partie des professions féminisées parce qu'elle recrute principalement dans des formations féminisées ; elle le serait donc encore davantage si les employeurs respectaient le ratio Femmes/Hommes des différentes filières éducatives parmi lesquelles ils recrutent. Il en est de même, en 2001, pour les assistant(e)s sociales et éducateurs, professions très féminisées dont le recrutement est marqué par une légère " préférence " des employeurs pour les candidats masculins, préférence qui disparaît sept ans plus tard. En 2008, la profession masculine des ouvriers agricoles s'ouvre à des recrutements féminins, en opposition à la forte ségrégation professionnelle d'origine éducative qui traduit une alimentation de la profession par des formations et niveaux d'enseignement à majorité masculine.

\subsection{Salaires et féminisation des professions}

Taux de féminisation du personnel et salaire moyen dans la profession sont-ils négativement corrélés ? Autrement dit, on cherche à savoir si plus il y a de femmes dans une profession, plus le salaire est bas.

L'écart de salaires moyens entre professions féminines et masculines se creuse sur la période ; en effet, le salaire moyen dans les premières passe de 1418 à 1519 euros, alors qu'il enregistre une évolution plus significative dans les professions masculines, passant de 1658 à 1902 euros.

Un raisonnement en termes de taux de croissance du salaire moyen sur la base des professions masculines et féminines occupées après dix ans d'expérience révèle que les hommes bénéficient d'une progression salariale de près de deux points supérieure à celle de leurs homologues féminines : $10,5 \%$ contre $8,7 \%$. Ces mouvements qui accentuent les disparités salariales entre hommes et femmes semblent donc corrélatifs d'un tassement de la ségrégation d'origine éducative, au profit de la ségrégation qui prend forme sur le marché du travail.

Ces écarts sont également liés aux différences de statut, la part des contrats à temps partiel étant plus importante parmi les femmes.

Le calcul du salaire moyen individuel sur l'ensemble des professions comptant un personnel féminisé à plus de $70 \%$, et en se limitant aux effectifs à temps plein, montre clairement que la rémunération y est plus faible qu'en moyenne et encore plus faible que dans 
les professions masculines : 1475 contre 1692 euros mensuels dans ces dernières (1 641 euros sur l'ensemble des professions) en 2001 et 1644 contre 1940 euros dans les professions masculines en 2008 (1900 euros sur l'ensemble des professions). Les occupants des professions féminisées ne sont guère mieux lotis si l'on raisonne en termes de salaire rapporté au niveau d'éducation; il s'agit ici de contrôler de possibles effets de composition liés à l'hétérogénéité des niveaux d'éducation selon les professions, ce qui revient à calculer le salaire moyen par année d'études dans la profession. Même si la hiérarchie des professions se modifie avec cet indicateur, les professions comportant une part importante de personnel féminin présentent là encore les salaires relatifs les plus bas.

Si l'on se réfère aux partisans de la théorie du capital humain, les professions féminisées seraient moins rémunératrices ; en effet, le capital humain mis en œuvre et les besoins en capital humain spécifique, c'est-à-dire la part de capital humain acquise chez un employeur spécifique et difficilement transférable d'un employeur à l'autre, y seraient moindres. Ces caractéristiques permettraient de limiter les répercussions négatives sur la carrière en cas d'interruptions d'activité, lesquelles seraient anticipées par les femmes pour fonder une famille et s'occuper des enfants ${ }^{7}$. S'agissant des dotations en formation initiale ou des formations suivies dans l'emploi pour les situations occupées au bout de dix ans de vie active, il apparaît que le personnel des professions féminisées n’est ni moins diplômé ni ne connaît un sous-investissement en formation dans l'emploi par rapport aux autres professions. Toutefois, on remarque que les femmes possèdent une expérience professionnelle effective de trois mois inférieure à ce qu'elle est dans les professions masculines ; on peut rapprocher ce différentiel du fait que les membres des professions féminines sont plus fréquemment parents que les autres : $67 \%$ d'entre eux sont déjà parent, d'au moins un enfant contre $55 \%$ des membres des professions mixtes et $50 \%$ des membres des professions masculines.

Pour illustrer de manière plus fine les liens entre ségrégation et évolutions salariales, examinons à présent ce qu'il en est pour cinq professions.

\section{Quelques professions emblématiques: 3 des infirmières aux policiers}

Les dimensions relatives à la ségrégation et au salaire vont être ici examinées pour trois professions fortement ségrégées. La première est très féminisée, il s'agit des infirmier(e)s. Les deux suivantes sont très masculinisées, l'une relevant du privé et l'autre majoritairement du public. Nous étudierons respectivement le groupe des ingénieurs de l'industrie, appartenant à la catégorie socioprofessionnelle des cadres et ingénieurs, et le groupe

7. On sait que les activités domestiques et familiales sont encore très largement à la charge des femmes (Ponthieux et Schreiber, 2006). 
des policiers, gendarmes et agents de sécurité, groupe de niveau employés. Enfin, pour faire pendant à ces professions ségréguées, les caractéristiques relatives à deux groupes professionnels mixtes sont analysées. Le premier groupe mixte, les professions intermédiaires commerciales, relève du secteur privé, alors que le second, constitué des enseignants du secondaire, certifiés ou agrégés, est rattaché pour l'essentiel au secteur public (cf. tableau 3).

\subsection{Les infirmières : un écart salarial en défaveur des femmes qui se creuse avec le temps}

Comme la majorité des professions occupées par les femmes dans le champ social et médical, la profession d'infirmière fait appel à des compétences considérées comme féminines : l'écoute, la capacité relationnelle, l'attention aux autres inhérente à la relation de soin sont autant de qualités supposées "naturelles " attachées à une identité "féminine " et souvent déconnectées du rapport salarial (Kergoat 1992). Infirmier(e) apparait donc comme un métier "de femme " car il semble requérir des "qualités [présentées comme] innées" chez les femmes ou qui "sinscrivent dans le prolongement des fonctions "naturelles" maternelles ou ménagères" (Perrot, 1987, pp. 3-4).

Déjà très féminisée en début de vie active, cette profession légalement règlementée se situe parmi celles dont la ségrégation professionnelle d'origine éducative est la plus élevée. Sa féminisation se poursuit en 2008 , en particulier du fait d'un renforcement de la ségrégation liée au marché du travail. À cette date, et parmi la cohorte des sortants en 1998 du système éducatif, elle compte près de $85 \%$ de femmes dans ses rangs. Les salaires moyens des hommes et des femmes sont assez proches, avec toutefois 80 euros d'écart mensuel à l'avantage des hommes après trois années de vie active. Mais cet écart a quadruplé en sept ans et les résultats des régressions économétriques aux deux dates témoignent de cette dégradation de la situation salariale des femmes, à conditions d'emploi, d'expérience et de formation égales : le fait d'être une femme abaisse le niveau de salaire de $14 \%$ après dix ans de carrière, pression à la baisse deux fois plus importante que sept ans auparavant. Cet accroissement de l'écart salarial entre les deux sexes tient pour partie aux différences de statut : l'exercice libéral, possible à partir de 2001 pour les diplômés de $1998^{8}$, est plus fréquemment choisi par les hommes que par les femmes. En 2005, 8 \% des infirmiers de cette génération pratiquaient leur activité dans ce cadre, lequel assure les progressions de revenu les plus importantes (Ferretti et Garrouste, 2008). Les salaires masculins progressent également davantage que les rémunérations des femmes du fait des spécialités exercées. Les hommes occupent en effet plus souvent des fonctions spécialisés (cadres infirmiers, en psychiatrie, en puériculture, en bloc opératoire, en anesthésie...) mieux rémunérées que les fonctions non spécialisées des soins généraux.

8. Le passage d'une activité salariée à libérale exige au moins trois années d'expérience professionnelle. 
À l'opposé des infirmier(e)s, on peut observer ce qu'il en est pour des groupes très masculinisés comme celui des professions de la sécurité ou à un niveau supérieur de qualification, le groupe des ingénieurs de l'industrie.

\subsection{Les professions de la sécurité : des disparités salariales qui s'estompent}

Le premier de ces groupes rassemble des emplois qui sont à la fois socialement associés à une image masculine et peu ancrés dans un cursus spécifique de formation initiale. Bien que s'inscrivant dans un contexte de quasi-stagnation du nombre de postes dans la fonction publique d'État, la demande sociale en termes de sécurité ne cesse de croittre, créant des opportunités pour des jeunes peu qualifiés (Simula, 2000). En conséquence, l'origine de la ségrégation tient essentiellement au recrutement des employeurs privilégiant largement une main-d'œuvre masculine. Pour autant, ce groupe professionnel donne à voir une évolution de la ségrégation qui tient aussi à des recrutements conformes à la composition sexuée des formations qui en constituent les viviers principaux : l'origine éducative de la ségrégation se renforce entre 2001 et 2008 ; cependant, elle reste mineure par rapport à la ségrégation qui se construit sur le marché du travail, privilégiant nettement la maind'œuvre masculine.

Basée sur la réussite à des concours, le recrutement des femmes au sein de la police nationale ou parmi les surveillants de prison, ou encore dans l'Armée depuis sa professionnalisation, a crû au cours de ces dernières décennies. Au cours du mouvement de féminisation de la police, les femmes qui l'intègrent tentent encore d'échapper aux stéréotypes de fragilité et d'indisponibilité, souvent en adoptant les codes virils en vigueur (Pruvost, 2007). Dans ce contexte et au sein de la Génération 98, avec l'accumulation d'expérience professionnelle, on assiste à l'homogénéisation des salaires masculins et féminins au sein de ce groupe professionnel : le différentiel de gains entre les sexes, en 2008, devient non significatif. Ce résultat peut laisser penser que les femmes persistant dans ces métiers "d'hommes " (elles sont un peu moins nombreuses après dix ans de vie professionnelle qu’en début de carrière) auraient réussi à " faire leur place " en rendant injustifiables les écarts de traitement salarial éprouvés initialement. Dans le cas spécifique de cette profession, les évolutions observées paraissent compatibles avec une explication en termes de discrimination statistique.

\subsection{Les ingénieurs de l'industrie : la ségrégation liée au marché du travail se renforce, les écarts de salaires importants s'accentuent}

Autre profession largement dominée par une main-d'œuvre masculine, celle des ingénieurs de l'industrie compte, en 2008, plus de $82 \%$ d'hommes.

Au-delà de ce secteur et de cette population, les fonctions de R\&D et la profession d'ingénieur en général restent massivement l'apanage des hommes même si, depuis le début 
des années 70, les femmes y sont devenues plus visibles (Marry, 2001 ; 2004). Reste que, parmi les ingénieurs de l'industrie, la proportion de femmes demeure encore limitée et les disparités de situation entre les sexes perdurent. La ségrégation se renforce entre 2001 et 2008, au détriment de la ségrégation héritée des formations alimentant ces professions et au bénéfice d'appariements sur le marché du travail qui privilégient une force de travail masculine. En conséquence, la contribution de la ségrégation liée au marché du travail à la ségrégation totale passe de 25 à près de $39 \%$. L'écart entre les salaires moyens par sexe est multiplié par cinq sur la période. C'est encore plus flagrant si l'on considère l'effet net relatif au fait d'être une femme, qui passe de 9 à $23 \%$ au bout de dix ans de vie active. Dans ces métiers à forte mobilité interne promotionnelle (Simonnet, Ulrich, 2009), on peut penser que les femmes éprouvent plus de difficultés à évoluer vers des fonctions managériales et à accéder aux postes à responsabilités.

Si les femmes sont moins souvent issues des écoles les plus prestigieuses, cette réalité n'explique qu'en partie l'augmentation des écarts de gains. Un autre élément d'explication relève de barrières à l'entrée (on parle aussi de " plafond de verre »), de nature discriminatoire, dont les femmes sont l'objet pour accéder aux plus hautes responsabilités hiérarchiques (Aeberhardt et Henriquez, 2005). Ainsi, en 2008, alors que $58 \%$ des ingénieurs de l'industrie masculins déclarent exercer des fonctions d'encadrement d'autres salariés, elles ne sont que $37 \%$ à avoir accédé à ce type de fonction.

\subsection{Les professions intermédiaires commerciales : l'expansion de la profession profite aux hommes, les écarts de salaires se creusent fortement}

Les professions intermédiaires commerciales restent des professions mixtes par excellence, mais la part des femmes baisse de 10 points avec le temps, au sein de la cohorte étudiée. C'est le résultat d'une ségrégation liée au marché du travail qui se renforce et qui s'oppose à la composante d'origine éducative en privilégiant les candidats masculins dans les recrutements. Le tassement de la composante éducative signe la diversification des recrutements, dont une partie s'opère au bénéfice de candidats munis d'une première expérience professionnelle acquise dans d'autres professions. Cette forte évolution de la composition sexuée, comparativement aux autres professions, s'explique aussi par le mode de gestion de la main-d'œuvre, fortement axé sur l'externe dans ces métiers où l'expérience, plus que le diplôme, concourt à la progression de carrière.

Ce résultat ne signe pas forcément une préférence affirmée des employeurs pour des commerciaux masculins ; il peut s'expliquer aussi par un détournement des femmes de ce type de profession marqué par des temps de travail élastiques et des déplacements fréquents dans certains cas; autant de conditions plus difficiles à concilier avec une vie de famille que celles d'un métier sédentaire aux horaires de travail maitrisés. De fait, les 


\section{Tableau 3}

Évolution de quelques groupes professionnels

\begin{tabular}{|c|c|c|c|}
\hline \multicolumn{2}{|l|}{ Groupe professionnel } & \multirow[b]{2}{*}{$\begin{array}{c}\text { Situation à } 3 \text { ans } \\
83,4 \\
0,345 \\
0,326 \\
0,018 \\
1481 \\
1445 \\
-6,69 \% \\
924\end{array}$} & \multirow[b]{2}{*}{$\begin{array}{c}\text { Situation à } \mathbf{1 0} \text { ans } \\
84,9 \\
0,360 \\
0,306 \\
0,054 \\
2313 \\
1893 \\
-14,14 \% \\
960\end{array}$} \\
\hline Infirmiers & $\begin{array}{l}\text { Part des femmes (\%) } \\
\text { Indice de ségrégation } \\
\ldots \text {... d'origine éducative } \\
\text {... liée au marché du travail } \\
\text { Salaire moyen des hommes à temps plein } \\
\text { Salaire moyen des femmes à temps plein } \\
\text { Effet net du sexe (\&) } \\
\text { Effectif du groupe }\end{array}$ & & \\
\hline $\begin{array}{l}\text { Professions intermédiaires } \\
\text { commerciales }\end{array}$ & $\begin{array}{l}\text { Part des femmes (\%) } \\
\text { Indice de ségrégation } \\
\ldots \text {... d'origine éducative } \\
\text {... liée au marché du travail } \\
\text { Salaire moyen des hommes à temps plein } \\
\text { Salaire moyen des femmes à temps plein } \\
\text { Effet net du sexe (\&) } \\
\text { Effectif du groupe }\end{array}$ & $\begin{array}{c}56,1 \\
0,072 \\
0,058 \\
0,013 \\
1345 \\
1309 \\
-5,10 \%^{*} \\
340\end{array}$ & $\begin{array}{c}46,4 \\
-0,024 \\
0,023 \\
-0,048 \\
2015 \\
1731 \\
-21,33 \% \\
403\end{array}$ \\
\hline Enseignants du secondaire & $\begin{array}{l}\text { Part des femmes (\%) } \\
\text { Indice de ségrégation } \\
\ldots \text {... d'origine éducative } \\
\ldots \text {.. liée au marché du travail } \\
\text { Salaire moyen des hommes à temps plein } \\
\text { Salaire moyen des femmes à temps plein } \\
\text { Effet net du sexe (\&) } \\
\text { Effectif du groupe }\end{array}$ & $\begin{array}{c}57,8 \\
0,089 \\
0,144 \\
-0,055 \\
1637 \\
1484 \\
-4,72 \%^{*} \\
242 \\
\end{array}$ & $\begin{array}{c}55,5 \\
0,066 \\
0,128 \\
-0,062 \\
2058 \\
2283 \\
\text { ns } \\
275\end{array}$ \\
\hline Ingénieurs de l'industrie & $\begin{array}{l}\text { Part des femmes (\%) } \\
\text { Indice de ségrégation } \\
\ldots \text {... d'origine éducative } \\
\ldots \text {.. liée au marché du travail } \\
\text { Salaire moyen des hommes à temps plein } \\
\text { Salaire moyen des femmes à temps plein } \\
\text { Effet net du sexe (\&) } \\
\text { Effectif du groupe }\end{array}$ & $\begin{array}{c}21,6 \\
-0,273 \\
-0,204 \\
-0,068 \\
2130 \\
2031 \\
-9,1 \% \\
203\end{array}$ & $\begin{array}{c}17,8 \\
-0,311 \\
-0,190 \\
-0,120 \\
3346 \\
2702 \\
-22,94 \% \\
231\end{array}$ \\
\hline $\begin{array}{l}\text { Police, Gendarmerie } \\
\text { Agents de sécurité, } \\
\text { Ambulanciers (Employés) }\end{array}$ & $\begin{array}{l}\text { Part des femmes (\%) } \\
\text { Indice de ségrégation } \\
\ldots \text {... d'origine éducative } \\
\ldots \text {.. liée au marché du travail } \\
\text { Salaire moyen des hommes à temps plein } \\
\text { Salaire moyen des femmes à temps plein } \\
\text { Effet net du sexe (\&) } \\
\text { Effectif du groupe }\end{array}$ & $\begin{array}{c}22,7 \\
-0,263 \\
-0,077 \\
-0,185 \\
1128 \\
1046 \\
-6,67 \% \\
209\end{array}$ & $\begin{array}{l}20,7 \\
-0,282 \\
-0,090 \\
-0,192 \\
1728 \\
1688 \\
\text { ns } \\
235\end{array}$ \\
\hline
\end{tabular}

Source : Enquêtes 2001 ; 2008 auprès de la Génération 98 . Champ : Jeunes en emploi. (\&) : Il s'agit du rendement salarial du fait d'être femme, toutes choses égales par ailleurs, obtenu par l'estimation - méthode des moindres carrés ordinaires (MCO) - d'une équation de salaire qui tient compte du secteur d'emploi, de la taille de l'entreprise, de sa localisation, du type de contrat et du temps de travail, des responsabilités hiérarchiques, de la durée et spécialité des études, ainsi que du fait d'avoir ou non au moins un enfant. On ne rapporte ici que les effets nets du sexe, significatifs au seuil de $5 \%$ (ou $10 \%$ s'ils sont signalés par une *), la mention «ns » indiquant un effet net non significatif. L'indice de ségrégation totale prend des valeurs dans l'intervalle $[0 ; 0,5]$ pour une cohorte équilibrée selon le sexe. La ségrégation est à son maximum pour la valeur 0,5 , ce qui signifie que la moitié de la population devrait changer de profession pour parvenir à un état de ségrégation nulle. Par convention, les signes négatifs indiquent que la part des hommes est supérieure à celle des femmes dans la profession et inversement. Remarquons que la somme en valeurs absolues des sous-indices peut dépasser l'indice de ségrégation totale dans la mesure où les deux types de ségrégation peuvent partiellement se compenser (opposition matérialisée par des signes opposés). Exemple de lecture : ainsi, la ségrégation s'accroît parmi les infirmiers en sept ans et dans le même temps, la composante éducative de cette ségrégation diminue au profit de la composante qui prend forme sur le marché du travail. 
jeunes femmes sont largement moins sur le "terrain », c'est-à-dire dans la représentation et la prospection, que les hommes (Cam, 2002).

Du point de vue des rémunérations, on sait que la mixité des études et des emplois n'est pas gage d'une plus grande équité salariale (Couppié et al., 2006). L'écart de salaire moyen a ici tendance à s'accroître sur la période alors que les salaires des femmes et des hommes sont de niveaux similaires en 2001. Cette évolution se traduit également au travers de l'effet du sexe sur les rémunérations lorsque l'on contrôle les conditions de formation, d'expérience et d'emploi.

La pénalisation salariale liée au sexe passe ainsi de 5 à plus de $21 \%$ en sept ans. Cette évolution trouve des explications dans les différences de fonctions exercées par les hommes et les femmes, toutes regroupées sous l'appellation de professions intermédiaires commerciales. Sur la base des intitulés des emplois occupés en début de carrière, les hommes agissent (agents commerciaux) et les femmes assistent (assistantes commerciales), les hommes représentent et négocient et les femmes animent ou visitent, comme le montre Cam (2002). Les fonctions de représentant en biens industriels ou de consommation forment le noyau dur des professions intermédiaires commerciales. Elles sont majoritairement détenues par les hommes, alors que les femmes se concentrent dans les emplois qui s’organisent autour de la vente des services, segment de la vente davantage accès sur la relation au client que sur les performances technologiques des produits (Cam, 2002). Ces disparités de situation sont donc pour beaucoup à l'origine des différences de salaire et de progressions salariales.

\subsection{Les enseignants du secondaire : des écarts de salaires non défavorables aux femmes}

Enfin, considérons une profession mixte, les enseignants du secondaire, mais dans laquelle les femmes restent prépondérantes sur la période (environ $56 \%$ au sein du personnel). Dans ce groupe, l'immense majorité des salariés relève du secteur public et les salaires y sont particulièrement homogènes selon le sexe. Pour les enseignants du secondaire au sein de l'Éducation nationale au moins, les titulaires des mêmes diplômes obtiennent en principe des positions et des salaires identiques, quelle que soit l'appartenance de sexe ; de même, leur avancement s'effectue à partir de critères neutres comme le succès au concours et l'ancienneté dans le grade. La ségrégation d'origine éducative, modérée, prédomine néanmoins au sein de cette profession ; mais son impact en faveur de recrutements féminins est contrebalancé par une composante de ségrégation liée au marché du travail orientée vers des recrutements masculins.

Ici comme dans nombre d'autres professions, la composante de la ségrégation, d'origine éducative, diminue avec les années de présence sur le marché du travail mais, phénomène moins fréquent, la composante liée au marché du travail n'augmente pas. L'écart salarial constaté en début de période - pénalité salariale de $5 \%$ pour les femmes - devient 
non significatif après dix années de carrière 9 . Globalement, les travaux sur les trajectoires professionnelles des enseignants sont rares et la mixité de la profession enseignante est beaucoup moins questionnée que celle des élèves (Cacouault, 1999 ; 2003). S'il existe des différences entre les disciplines enseignées par les hommes et les femmes, ces dernières sont aussi plus souvent mariées et mères de famille (68 \% ayant déjà au moins un enfant contre $61 \%$ des hommes enseignants du secondaire); cela pourrait les rendre moins enclines à demander des mutations et à candidater aux fonctions de direction d'établissement.

Au total, hormis les cas du groupe professionnel des enseignants du secondaire - qui échappe le plus nettement au marché concurrentiel - et des emplois de la sécurité, la situation salariale des femmes rapportée à celles des hommes tend à se dégrader dans les autres professions ; dans le même temps, la part de la ségrégation d'origine éducative s'affaiblit dans la construction de la ségrégation professionnelle observée sur le marché du travail. Les emplois de la sécurité (hors responsables) constituent le seul groupe pour lequel la ségrégation d'origine éducative ne faiblit pas; c'est aussi l'un des deux seuls groupes pour lesquels l'écart de salaire disparaît au fil de l'expérience.

\section{Conclusion}

Le regard porté ici sur quelques professions laisse présumer que les liens entre ségrégation professionnelle, niveaux de salaire et écarts de salaire entre hommes et femmes ne sont pas univoques. Les disparités salariales ont ainsi tendance à s'estomper dans un milieu très masculin comme celui des emplois de la sécurité (au niveau employés). À l'inverse, parmi les ingénieurs de l'industrie, les progressions de carrière semblent en moyenne nettement plus poussives pour les femmes. Cependant, globalement, c'est-à-dire à l'aune des 77 groupes professionnels distingués, la ségrégation héritée de la composition sexuée des filières éducatives s'affaiblit et les disparités de salaire entre hommes et femmes s'accroissent.

Notre objectif n'était pas ici de tester la portée empirique des explications théoriques évoquées au début; notre travail suggère néanmoins quelques diagnostics. Dans la plupart des groupes professionnels, l'écart salarial entre hommes et femmes n'a pas de caractère transitoire, au contraire ; déjà présent en début de vie active, cet écart se creuse, dans la majorité des professions, après dix années passées sur le marché du travail. Cela tend à infirmer une interprétation théorique générale en termes de discrimination statistique qui expliquerait l'existence d'une pénalité salariale en début de vie active à l'encontre des femmes du fait des incertitudes pesant sur leur productivité réelle dans l'emploi, pénalité

9. La grande majorité des emplois d'enseignants du secondaire relève du secteur public, régi par des conditions de recrutement et de rémunération a priori neutres du point de vue du genre. Dans ces conditions, l'écart de salaire (en 2001) peut s'appuyer sur des distinctions et des pratiques que l'enquête ne permet pas de prendre en compte ; ainsi, la répartition certifiés / agrégés est potentiellement sexuée ; de même, le nombre d'heures supplémentaires effectuées par les unes et les autres est une source possible de différenciation des rémunérations. 
qui s'effacerait progressivement une fois qu'elles auraient « fait leurs preuves ». Les explications fondées sur l'approche du capital humain, qui verrait les professions très féminisées comme des professions où les investissements en capital humain seraient moins exposés à des risques de dépréciation en cas d'interruptions en cours de carrière, ne sont pas non plus convaincantes ; même s'il existe une corrélation entre le fait d'occuper un emploi dans une profession féminine et le fait d'être devenu parent sur la période, à individu de sexe donné.

Reste les autres interprétations qu'il conviendrait d'aborder de manière systématique, notamment celle de la dévaluation des compétences dans les professions féminines. Un tel projet devrait alors s'appuyer sur les enseignements des analyses fines des contenus d'emploi, telles que celles réalisées par Lemière et Silvera (2010). Il serait également pertinent d'identifier d'éventuels comportements d'auto-sélection des femmes sur le marché du travail, comme le suggère l'approche par les différences compensatrices. Cette approche met en avant l'existence d'autres critères de désirabilité des emplois que le niveau de salaire ; critères qui conduisent les femmes, davantage que les hommes, à privilégier certaines situations professionnelles comportant, de leur point de vue, un certain nombre d'avantages non monétaires (sécurité de l'emploi, possibilités de concilier temps familiaux et temps professionnels, etc.) au détriment du niveau de rémunération.

Nos résultats témoignent de l'intérêt de prendre en compte la profession comme une unité contextuelle pertinente. Dans des travaux futurs, il conviendra de poursuivre cette logique d'analyse ; de même, il serait pertinent d'apprécier plus systématiquement le rôle des composantes de la ségrégation professionnelle sur le salaire, tout en contrôlant un certain nombre de dimensions qui leurs sont potentiellement corrélées, telles que le statut des emplois, le prestige des professions, le taux de déclassement..., et qui influencent le niveau des rémunérations.

\section{Bibliographie}

Aeberhardt R. et Horacio Henriquez H. (2005), «Ingénieurs diplômés : leur salaire en 2004 ", Insee Première, n 1054, décembre.

Aigner D.J. and Cain G.G. (1977), "Statistical Theories of Discrimination in Labor Markets”, Industrial and Labor Relations Review, Vol. 30, n 2, pp. 175-187.

Anker R. (1998), Gender and Jobs. Sex Segregation and Occupations in the World, Geneva: International Labour Office.

BAKer M. and Fortin N. (2001), "Occupational gender composition and wages in Canada, 1987-1988”, Canadian Journal of Economics, Vol. 34, n² 2, pp. 345-376.

Bielby W.T. and Baron J.N. (1986), "Men and Women at work: sex segregation and statistical discrimination", American Journal of Sociology, Vol. 91, pp. 759-799. 
Bayard K., Hellerstein J., Neumark D. et Troske K. (2003), “ New evidence on sex segregation and sex differences in wages from matched employee-employer data", Journal of Labor Economics, Vol. 21, n 4, pp. 887-922.

Becker G.S. (1971), The Economics of Discrimination (2 ${ }^{\text {nd }}$ edition), University of Chicago Press, Chicago.

Busch A., Holst E. (2011), "Gender-specific occupational segregation, glass ceiling effects and earnings in managerial positions: results of a fixed effect model", IZA Discussion Paper n ${ }^{\circ} 545$.

Cacouault-Bitaud M. (1999), "Professeur du secondaire : une profession féminine ? Eléments pour une approche socio-historique », Genèses, n 36, septembre, pp. 92-115.

Cacouault-Bitaud M. (2003), "La sociologie de l'éducation et les enseignants : cherchez la femme ", in Laufer J., Marry C., Maruani M., Le travail du genre. Les sciences sociales du travail à l'épreuve de la différence des sexes, Paris, La Découverte, collection "Recherches ", pp. 163-180.

CAM P. (2002), Les intermédiaires du commerce : métiers masculins, métiers féminins, in Quand les jeunes entrent en emploi, ouvrage coordonné par M. Arliaud et H. ECKerT, Paris, La Dispute.

Cockburn C. (1991), In the way of women. Sex resistance to sex equality in organizations, Ithaca, N.Y.:ILR Press.

Cohen P.N. and Huffman M.T. (2003), "Individuals, jobs and labour markets: the devaluation of women's work", American Sociological Review, 68, pp. 443-463.

Couppié T., Epiphane D. (2004a), "Des bancs de l'école aux postes de travail... Chronique d'une ségrégation annoncée ", Céreq, Notes Emploi Formation, 6.

Couppié T. et Epiphane D. (2006), «La ségrégation des hommes et des femmes dans les métiers : entre héritage scolaire et construction sur le marché du travail ", Formation Emploi, n 93, pp. 11-27.

Couppié T., Epiphane D. (2009), “Allongement séculaire des scolarités féminines et évolution récente de la ségrégation dans les professions : quelles relations ? ", Economie et Société, AB, n³0, pp. 87-122.

Couppié T., Dupray A. et Moullet S. (2006), " Les salaires des hommes et femmes en début de vie active : des sources de disparité variables selon les professions ", Formation Emploi, n 93, pp. 29-47.

Couppié T., Dupray A. et Moullet S. (2011), " Les effets de moyen-terme de la ségrégation professionnelle selon le genre sur le salaire des hommes et des femmes ", in Béduwé et al. (eds.), Les nouvelles ségrégations scolaires et professionnelles, Céreq, Relief, $\mathrm{n}^{\circ}$ 34. pp. 331-356. 
DAUne-Richard A-M. (1998), "Qualifications et représentations sociales », in J. LAUfER, Maruani M. et Marry C. (dirs.), Les nouvelles Frontières de l'Inégalité. Hommes et Femmes sur le Marché du Travail. Paris, La Découverte, pp. 47-58.

England P. (1982), "The Failure of Human Capital Theory to Explain Occupational Sex Segregation”, Journal of Human Resources, vol. 17, pp. 358-70.

England P. (1992), Comparable Worth. Theory and Evidence, New-York: Aldine.

England P., Farkas G., Kilbourne B.S. and Dou T. (1988), "Explaining occupational sex segregation and wages: findings from a model with fixed effects", American Sociological Review, vol. 53 (4), pp. 544-558.

Epiphane D., Gasquet C., Moullet S. (2008), «Quels sont les liens entre trajectoires d'emploi et situation familiale?" in J.-J. Paul, J. Rose (dirs.) Les relations formationemploi en 55 questions, Dunod, pp. 262-267

Ferretti C., Garrouste C. (2008), « Les sept premières années de carrière des infirmiers diplômés en 1998 ", DREES, Études et Résultats, n 671.

FILER R. K. (1983), "Sexual differences in earnings: the role of individual personalities and tastes", The Journal of Human Resources, 18, pp. 82-98.

Groshen E. L. (1991), "The structure of the female/male wage differential: is it who you are, what you do or where you work?", The Journal of Human Resources, vol. 26, pp. 457-472.

Hansen J. et Wahlberg R. (2008), "Occupational gender composition and the gender wage gap in Sweden”, Research in Labor Economics, Vol. 28, pp. 353-369.

Kergoat D. (avec la collab. de Imbert F.) (1992), Ouvrières et infirmières : deux rapports à la qualification, Lamare, Paris.

Killingsworth M. R. (1987), "Heterogeneous preferences, compensating wage differentials, and comparable worth", Quarterly Journal of Economics, 102, pp. 727-742.

Lemière S. et Silvera R. (2010), "Un salaire égal pour un travail de valeur comparable entre les femmes et les hommes. Résultats de comparaisons d'emplois ", Revue de l'IRES, 66/3, pp. 63-92.

Macpherson D.A. and Hirsch B. (1995), "Wages and gender composition: why do women's jobs pay less?”, Journal of Labor economics, Vol. 13, n .3, pp. 426-71.

Marry C. (2004), Les femmes ingénieurs, une révolution respectueuse, Belin, coll. "Perspectives sociologiques".

MARRY C. (2001), «La féminisation de la profession d'ingénieur : une comparaison FranceAllemagne ", in Bouffartigue P. (ed.), Les cadres. La grande rupture, La Découverte, pp. 281- 296. 
Meng X. et Meurs D. (2001), «Différences de structure des emplois et écart salarial entre hommes et femmes en France ", Economie et Prévision, n 148, pp. 113-126.

Mincer J., Ofex H. (1982), "Interrupted work careers: depreciation and restoration of human capital", The Journal of Human Resources, vol. 17, pp. 3-24.

Оква M. (2004), "L'accès des femmes aux métiers ", Dares, Premières Informations et Premières Synthèses, $\mathrm{n}^{\circ} 31.2$.

Perrot M. (1987), "Qu'est-ce qu’un métier de femme ? ", Le Mouvement social, n 140.

Ponthieux S., Schreiber A. (2006) "Dans les couples salariés la répartition du travail domestique reste inégale”, Insee, Données Sociales, pp. 43-51.

Petersen T. and Saporta I. (2004), "The opportunity structure for discrimination", American Journal of Sociology, vol. 109, pp. 852-901.

Polacheк S.W. (1981), "Occupational self-selection: a human capital approach to sex differences in occupational structure", The review of Economics and Statistics, 63(1), pp. 60-69.

Pruvost G. (2007), Profession : policier. Sexe : féminin, Éditions de la MSH, Paris.

Reskin B. F. (1993), "Sex segregation in the workplace", Annual Review of Sociology, 19, pp. 241-270.

de Rujuter J.M.P. et Huffman M. (2003), "Gender composition effects in the Netherlands: a multilevel analysis of occupational wage inequality”, Social Science Research, Vol. 32, pp. 312-334.

de Ruijter J.M.P., van Doorne-Huiskes A. et Schippers J.J. (2003), "Size and cause of the occupational gender wage-gap in the Netherlands", European Sociological Review, vol. 19, pp. 345-360.

Simonnet V., Ulrich V. (2009), "La mobilité entre métiers », Dares, Premières Informations et Premières Synthèses, janvier, $\mathrm{n}^{\circ} 05.3$.

Simula P. (2000), "La dynamique des emplois dans la sécurité ", Etudes et recherches, Institut des Hautes Etudes de la Sécurité Intérieure.

Sorensen E. (1990), "The crowding hypothesis and comparable worth issue: a survey and new results", Journal of Human Resources, vol. 25(1), pp. 55-89.

Steinberg R.J. (1990), "Social construction of skill. Gender, power and comparable worth”, Work \& Occupations, vol. 17(4), pp. 449-482.

Tomaskovic-Devey D. and Skaggs S. (2002), "Sex segregation, labor process organization and gender earnings inequality”, American Journal of Sociology, 108, pp. 102-128. 